\title{
A cilia-inspired closed-loop sensor-actuator array
}

\author{
James G.H. Whiting, Richard Mayne, Chris Melhuish, Andrew Adamatzky \\ Unconventional Computing Centre and Bristol Robotics Laboratory, University of the \\ West of England, Bristol, UK
}

\begin{abstract}
Cilia are finger-like cell-surface organelles that are used by certain varieties of aquatic unicellular organisms for motility, sensing and object manipulation. Initiated by internal generators and external mechanical and chemical stimuli, coordinated undulations of cilia lead to the motion of a fluid surrounding the organism. This motion transports micro-particles towards an oral cavity and provides motile force. Inspired by the emergent properties of cilia possessed by the pond organism $P$. caudatum, we propose a novel smart surface with closed-loop control using sensor-actuators pairings that can manipulate objects. Each vibrating motor actuator is controlled by a localised microcontroller which utilises proximity sensor information to initiate actuation. The circuit boards are designed to be plug-and-play and are infinitely up-scalable and reconfigurable. The smart surface is capable of moving objects at a speed of 7.2 millimetres per second in forward or reverse direction. Further development of this platform will include more anatomically similar biomimetic cilia and control.
\end{abstract}

Keywords:

Bioinspiration; Artificial Cilia; Object Manipulation; sorting platforms.

\section{Introduction}

Cilia are finger-like cellular projections possessed by a wide variety of eukaryotic cell types; there is a high degree of ciliary homology between different species ${ }^{1}$. In multi-ciliated cells, these organelles possess the ability to beat with a whip-like motion ${ }^{2]}$ : this primarily serves to provide motile force in unicellular organisms (ciliated protozoa and algae) ${ }^{3}$ or otherwise drives fluid movements in multicellular organisms, such as the mucociliary escalator in 


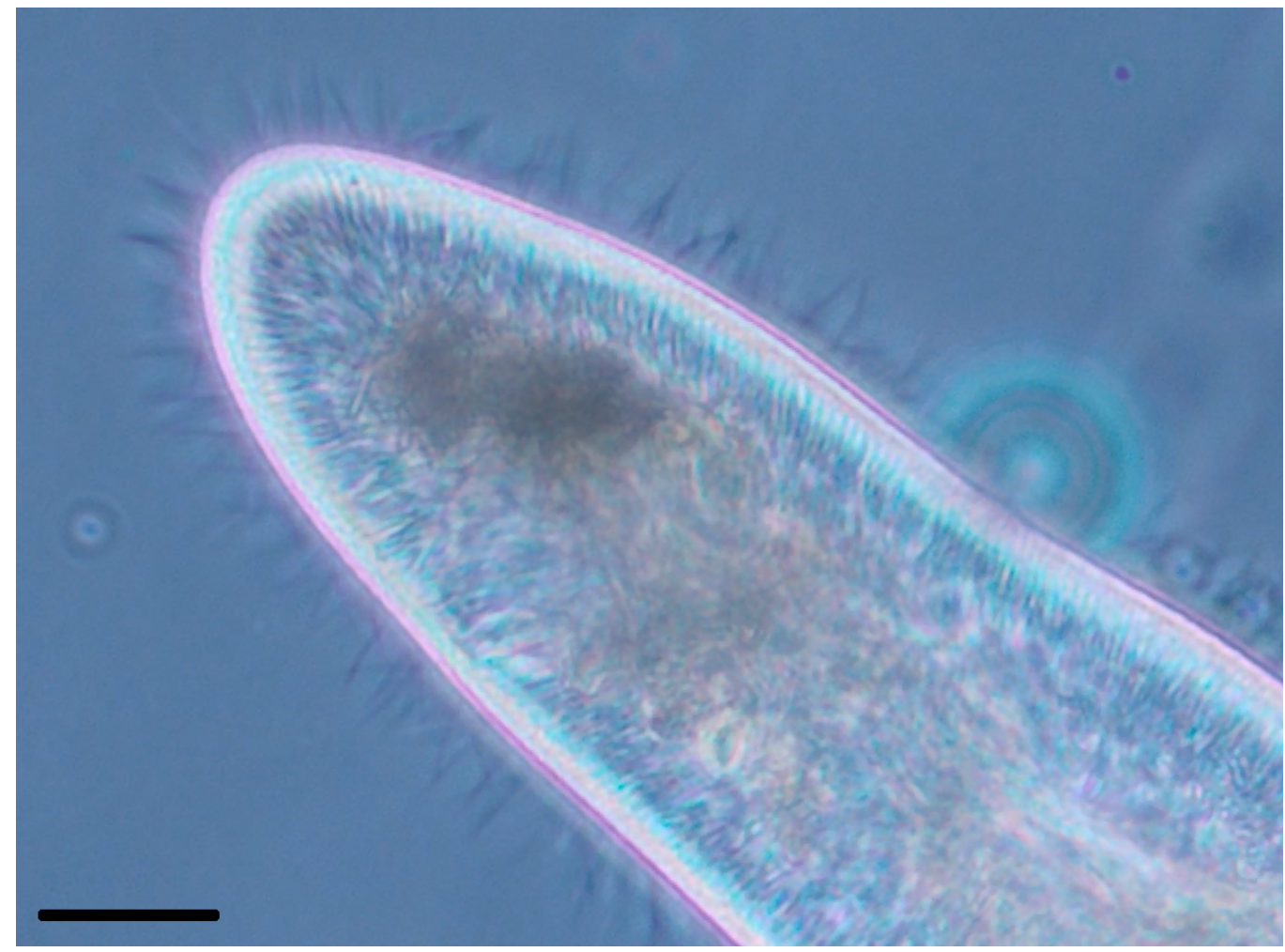

Figure 1: Phase contrast micrograph of $P$. caudatum's anterior apex. Cilia may be observed coating the organism. Scale bar $25 \mu \mathrm{m}$.

human bronchial epithelial tissues [ []ㅡ. Secondary roles include manipulation of proximate particulate matter, sensing and substance absorption ${ }^{[5 / 3 / 4}$.

As each cilium is a discrete sensing element and actuator in its own right; a ciliated cell may be viewed as a parallel actuator array with decentralised control. Crucially, the ability of ciliated cells for spontaneously generating metachronal waves, sequential travelling wave patterns of ciliary beating, demonstrates the capacity these systems possess for facilitating emergent behaviour in the absence of a centralised control system. Hence, cilia arrays are a prime target for the fabrication of bio-inspired sensorial-actuation systems; the investigation detailed here is presented in context with our aims to develop a smart actuation surface inspired by the cilia possessed by the protozoan P. caudatum.

Object manipulation surfaces in industry are predominantly conveyor belt systems as they are very reliable due to simplicity and autonomy due to the 
direction of the belt; this arrangement has been used for hundreds of years ${ }^{677}$. Modern conveyor belt systems are more complex than their predecessors by having camera sensing and sorting actuators however still have the basic motorised belt for manipulation; a shortfall of having belt fed conveyors is the lack of adaptability to new sorting or manipulation tasks, with the system requiring redesign to change the manipulation direction and protocol. Belt fed conveyors are also unable to manipulate multiple objects in different directions simultaneously and are often without sensor feedback.

In devices with open-loop control, the actuation is sensor-less and without feedback. In object manipulation applications, the processor does not receive any explicit information about the object's geometry, and actuation is based solely on interaction of the geometry of the resulting force field and the geometry of the transported object ${ }^{89}$. Open-loop control utilises a methodology of pre-programmed manipulation, which are calculated by a computer and generated on a surface of the manipulator; open-loop systems are often used for transport rather than specific manipulation tasks such as sorting. Closed-loop control is sensory, with feedback of an important parameter for error-resistance and improved accuracy. In systems of this type, sensors inform the central processor about the manipulated object at each moment of time. The processor changes patterns of actions depending upon the particulars of the object motion. The information about the manipulated object could be provided either by an external sensor, such as a vision system, or by a distributed sensing (optical or tactile) system, i.e., when every actuator of the array has its own sensor.

Alternatives to conveyor belt systems are present in industry, such as vibratory bowl feeders or sensor-less mechanical systems 10111. Mechanical design coupled with a directional motile force, from either vibration or conveyor systems, manipulates objects in an open loop system; the design of these systems is very part specific, with each part often requiring their own system to orientate an object. It is the aim of this project to create a platform which is not object specific and can be adaptable.

Most of the work on sensor based parallel manipulator devices has employed centralised control and has been implemented at the micro-scale such as micro-electro-mechanical systems (MEMS) and at the macro-scale with vibrating plates 9 . These MEMS or vibrating plates move objects by creating a force vector via their actuation mechanism. The idea of centralised control implies that the device has a central processing system, which receives and analyses the information from micro-actuators or object position. Planned 
manipulation with programmed force vectors is a hard computational task. The problem becomes even more resource-demanding when manipulating multiple objects. Furthermore, programmed fields, in their 'pure' form, offer a narrow space for manoeuvre as they have a limited number of combinations of 'typical' fields that successfully process an object 12113. Centralised control, applied to parallel manipulating systems is also a drawback from the points of view of system scaling, maintenance, reliability and compactness. In view of the discrete nature of the manipulator and disadvantages of centralised control, the idea of decentralised control, with use of non linear media is promising.

With a little increase of communication complexity we can couple actuating units with their own unique sensors and with their nearest neighbours and thus not simply transfer information between units but collectively produce a co-ordinated action using local information. In this way local sensing and actuation contributes to a global emergent outcome, even in the absence of a centralised controller. Such an approach is complementary to the structure and function of cilia.

Alternative manipulation surfaces have been proposed which document the development of smart surfaces; micro-actuator arrays and micro-electricalmechanical systems (MEMS) based systems have been proposed ${ }^{[14}$; dealing with small scale object manipulation, however many are based on single control with optical feedback using camera-computer systems $15[16 \mid 17$ rather than distributed control, or even open loop control without sensors $18 / 19$. These closed loop systems offer a greater degree of accuracy than traditional conveyor belt systems but lack distributed autonomy and to some degree scalability.

This paper investigates the feasibility of an entirely up-scalable, plug-andplay, sensor-actuator system with localised autonomy using vibrating motors for object manipulation. Investigating a bio-inspired control mechanism, we test the level of object manipulation of a closed loop protocol similar to that of the $P$. caudatum organism.

\section{Methods}

\subsection{Hardware}

The plug-and-play boards are based on open source project boards $(20 \times 10$ centimetres) which are designed for cellular automata software and control 
ultrabright LEDs using pulse-width-modulation (Octolively Kit ${ }^{1}$ ). These boards have been adapted to control low power vibrating motors with custom software. Each board is controlled by an ATmega164P microcontroller, there are 8 proximity sensors and 8 vibration actuators per board (each uniquely paired); proximity sensors are $940 \mathrm{~nm}$ infra-red phototransistors, which have programmable sensitivity. The IR phototransistors detect the presence of an object above them by surface reflection from corresponding IR LEDs and the actuator is activated accordingly by way of increased RPM. It is possible to re-programme this control system to perform different tasks when objects are detected, or indeed replace the IR sensors with something different which could be task dependent. Reversing of direction in this application was changed in the software however it is entirely possible that direction reversal could be triggered by another parameter; different sized or shaped objects could be manipulated differently in order for them to be sorted into different categories. The actuators are Precision Microdrives $31010 \mathrm{~mm}$ diameter vibrating DC motors ${ }^{2}$, A total of 6 boards are tiled to produce a $6 \times 8$ matrix of actuators. The motors are mounted on the PCB using medium density PVC foam $5 \mathrm{~mm}$ square and $9.6 \mathrm{~mm}$ high pads (RS Components, UK) to allow adequate spacing above the other components while maintaining a degree of flexibility for vibration. The motors are capable of rotating at 12200 RPM, the equivalent of approximately $203 \mathrm{~Hz}$. The motors create vibration by rotating an offset mass around the shaft; the typical vibration amplitude is 1.3 G. This rotating is similar to cilia in P. caudatum in that the cilia rotate at an offset angle, creating a directional force in the surrounding fluid; the similarity of the reversible rotation and trajectory of the distal tip of the cilia is also notable.

\subsection{Software}

In vivo, Paramecia's cilia beat constantly; changes in beating frequency are caused by external stimuli that precipitate calcium ion influx/efflux via membrane hyper-polarisation (which causes an increase in frequency) or depolarisation (decrease) ${ }^{20}$. An example of such an external stimulus is the detection of food; a favourable chemo-attractant gradient detected in the local environment results in changes in beating frequency, however the direction of

\footnotetext{
1 http://wiki.evilmadscientist.com/Octolively

${ }^{2}$ https://www.precisionmicrodrives.com/product/310-103-10mm-vibration-motor-3mm-type
} 
ciliary beat appears independent however beating direction may reverse when an unfavourable stimulus depolarises the ciliary membrane to the degree that calcium-permeable voltage-gated ion channels open. Therefore it can be assumed that beating frequency modulation is instrumental in facilitating both the positive and negative taxes with favourable and unfavourable conditions, respectively. Our material approximation of this system is to have the cilia moving slowly until the surface is stimulated. When stimulation occurs, the organism increases beating frequency thus moves faster or manipulates an object with more speed. To mimic this control mechanism with the vibrating motors, each motor is programmed to rotate slowly, until such point that the local sensor detects an object above the actuating motor, in a closed loop system; the fast beating speed is the full RPM of the motor. No neighbour communication between cilia is currently required at this stage however local communication is being investigated, as the platform allows for neighbour data-links.

\subsection{Experimental set up}

The object which is used to test the manipulation is a piece of square cardboard $12 \mathrm{~cm}$ long, $1.5 \mathrm{~mm}$ thick and weighs 10 grams; the size and shape of the object ensured that several cilia were always in contact with the disc at any time. The tiled boards were placed on a flat and even platform to ensure no gravitational bias. The motors can be driven in either direction, so in order to control manipulation, the motors were driven in both clockwise and anticlockwise direction to observe the effect of different eccentric mass rotation. A HP 2300 USB Webcam was used to record movement and rotation of the object at a frequency of 15 frames per second at SVGA resolution. The object was coloured red to facilitate tracking. Capture and analysis of the video was performed in Matlab R2016a software and measured the object's position in each frame and calculated the overall movement during the measurement period. The object was identified in the webcam due to its unique colour in the camera's field of view; the webcam was placed directly above the boards so that only the platform is visible in the camera's video, equating to 2 pixels per millimetre. Each rotation direction test was repeated a total of 12 times to facilitate a sufficiently large sample size for descriptive statistical analysis. An example screen shot from the camera while tracking the object is shown in figure 2; the outline of the object was identified and the centre mass is calculated by the custom software and pixel position was superimposed on the image displayed to the user. The repeat experiments were performed whereby 


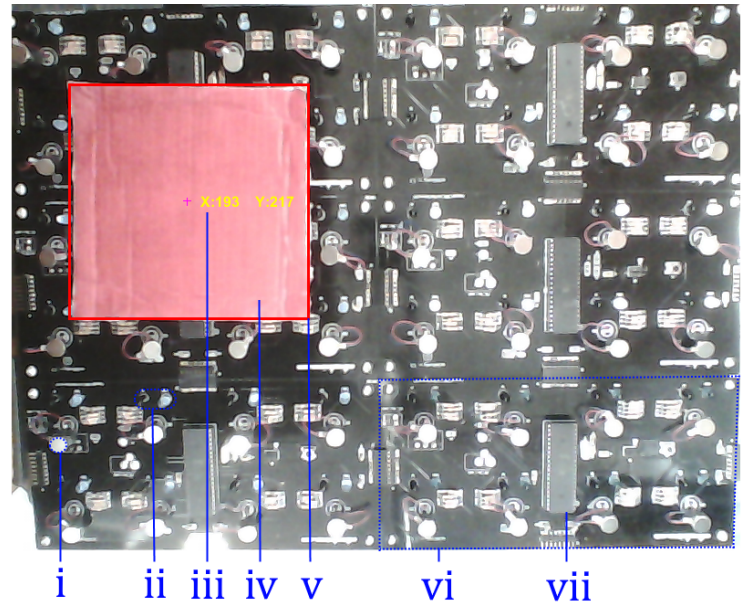

Figure 2: An example image from the overhead camera and Matlab software with the tiled PCBs in the field of view, together with the tracking details overlaid on the object where i) is a vibrating motor, ii) a pair of IR LEDs and IR phototransistors, iii) centre mass of the red object with appropriate coordinates, iv) the red card object being manipulated, v) the outline of the red object, vi) one plug-and-play board, vii) an ATmega164P microprocessor

the object was placed at random on the sensor-actuator surface and the smart surface manipulated the object with the closed-loop autonomous algorithm described in section 2.2, 12 repeats were performed and the movement of the object was tracked via the webcam and the location per frame was recorded.

\section{Results}

Figure 3 shows an example of the movement an object over a period of approximately 25 seconds; the image shows the start and stop positions and the blue cross shows the centre of mass of the object in each frame. The objects were often rotated by the motors in addition to moving over the surface; the centre of mass was used to track the object as this was a better method of position detection and rotation of the object is not relevant for object locomotion and sorting. The object appears to move outside the platform on the example which is elevated above the table so not to interfere with an object moving near the edge of the platform; if the object fell from the edge of the platform then tracking ceased. Starting positions were spread out in order to evaluate the whole surface's manipulation ability.

Figure 4 shows the distance and angle of movement for each of the 12 


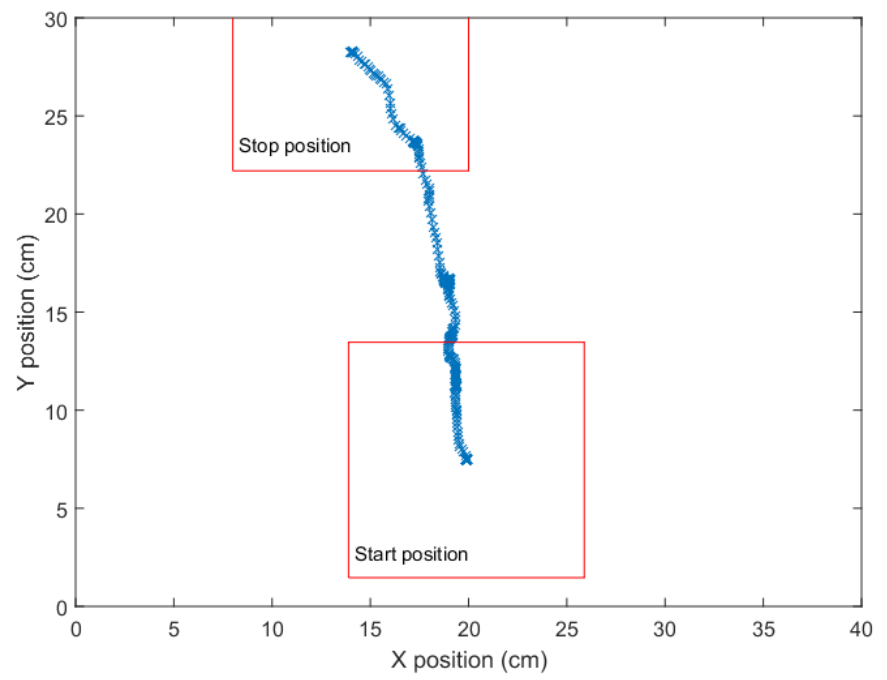

Figure 3: An example of the movement from the start to stop position tracking of the object. The object's centre-mass is represented by the blue crosses (one per frame) and the red squares represent the start and stop positions

repeats; the object occasionally slowed or moved back and forth throughout tracking, which after a period of a few seconds returned to original direction and speed. As is demonstrated in figure 3 , the object did not always travel in a linear fashion due to the constant shift in relative actuator-object positioning; to facilitate comparison between repeats, the speed vectors were calculated.

It is clear from figure 4 that the direction of all the repeats for a given motor polarity was similar, with normal polarity the object moved between North and North-East, with a mean direction was 29.8 degrees (standard deviation 15.1 degrees); using reversed motor polarity direction of movement was in the South-South-East to South-West, with the mean direction was 212.8 degrees with a standard deviation of 19.2 degrees. Calculating the speed for normal polarity repeats equates to a mean object speed of 7.1 millimetres per second, with a standard deviation of 2.1 millimetres per second; with motors in reversed polarity the mean object speed is 7.5 millimetres per second with a standard deviation of 2.3 millimetres per second. There was no statistical difference between the speed of the object when using normal or reversed motor polarity using a two tailed t-test $(\mathrm{p}=0.6596)$, while there was 
0

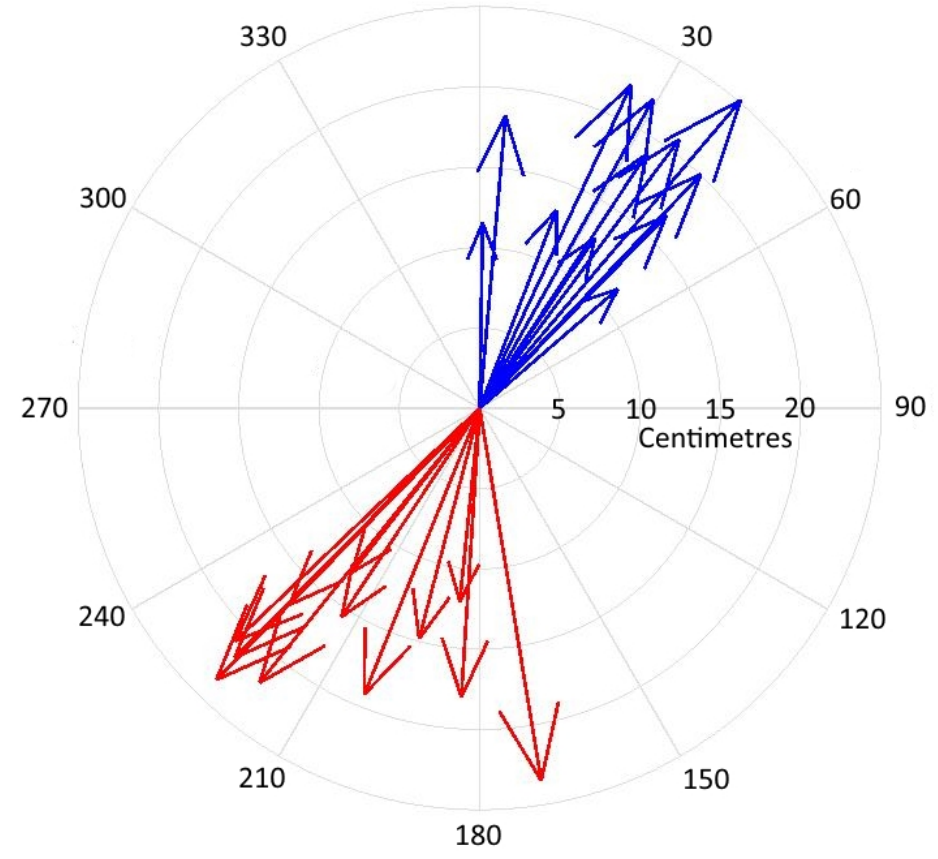

Figure 4: Direction vector plot for both motor polarities, each with 12 repeats. Vector magnitude represents distance moved and vector angle represents overall direction of movement. Red vectors represent reverse motor polarity while blue vectors represent normal motor polarity. 
a significant difference between direction in which the object moved using a two tailed t-test $(\mathrm{p}<0.01)$.

\section{Discussion}

The results demonstrate that the smart surface is capable of sensing and moving an object placed on the cilia bio-inspired surface by IR light reflection from the object and resultant local vibratory motor force. The objects are transported across the surface in a North to North-Easterly direction with normal motor polarity and South-South-East to South-West with reversed motor polarity. This demonstrates that we have 2 directional transportation using this system, the manipulation equivalent of reversing the direction of the conveyor belt; since each vibrating motor can be controlled individually, local areas of reversed direction can be created, meaning unlike conveyor belts, objects on the same surface can travel in opposite directions. In addition to being transported, objects were rotated as well as transversely moved across the surface, this was the result of the motor rotation; the necessity or importance of object orientation is application dependent. Some applications such as object sorting do not require orientation manipulation, however the feasibility of controlled rotation is the subject of future work using this system now that a re-programmable sensor-actuator platform has been developed. While the same square object was used in a testing environment, the authors also tested other shaped objects with no apparent difference in object manipulation; so long as an object is large enough to sit atop at least 3 motors at all times, it will be manipulated in the same direction although it may face more or less rotation while it is being moved.

This mode of bidirectional eccentric motor actuation is phenomenologically similar to the beating of $P$. caudatum cilia, as all object manipulation is unidirectional (anterior to posterior, with a constant counter-clockwise rotation) as the organism swims forwards (and vice versa when the organism reverses its direction of ciliary beating). The biological purpose of this mechanism is, as aforementioned, to facilitate grazing and drive net anterior or posterior migration, as these movements create fluid vortexes about the organism.

Previous smart surfaces or artificial cilia platforms are open-loop systems ${ }^{18 \mid 19}$ for which there is no sensor-actuator control; without any feedback they would have little control over the object let alone knowledge of the object's position. Some smart surfaces employ control and feedback via 
a computer and camera 15/16/17, where the computer interprets the object's position on the surface using the camera as a single sensing agent and manipulates the actuators with closed-loop control. Computer-camera closed-loop systems offer an improvement over open-loop systems however the subject of scalability becomes issue; the camera's view needs to be modified for every change in smart surface layout, potentially with the need for calibration, while the computer's control of an increasing number of sensors becomes ever more demanding. In this experiment the camera is used purely for data capture and analysis, it offers no feedback to the system. The system presented here offers only local sensor-actuator control, which is a significant improvement for scalability and reconfiguration, as the boards can be arranged in any shape, without the need to modify the feedback or control mechanism. The board layout also supports local communication between boards and sensoractuator units so different algorithms can be implemented with neighbour communication should this be required. This integration of a sensing loop into the platform represents the key novelty of our approach which, crucially, is designed to mimic the properties of biological cilia. While it would be possibly to shrink the boards down for scaling down the boards, there is significant advantage of the up-scalability of plug and play boards; the lack of centralised control means that absolutely no software reconfiguration needs to occur to increase the number of boards (and thus size of the platform) or change the overall shape of the platform.

Closed loop systems allow for more predictive manipulation. Governed by certain mapping rules, the closed loop system can fulfil the function of a simple conveyor, where the direction of motion and the final destination of the moved object depend on its initial displacement and orientation on the grid. The closed loop manipulators are sensitive to an objects shape. The manipulators are re-programmable, decentralised geometrical filters or sorting machines. Studies of parallel manipulators revealed beneficial properties and a high potential for distributed and decentralised object processing at micro and macro scales.

A further direction for study would be the emulation of various emergent properties exhibited by $P$. caudatum cilia, such as their spontaneous alternation in beating direction in response to an unfavourable stimulus exhibited by Paramecia resulting in reversed direction of travel. Integration of this function would allow for modulation of transport direction based on some sensed property of the objects moving over it. Another biological phenomenon that could be implemented as a method of cilia control is that of metachronal 
waves, which is presumably a route towards more efficient transport. As the mechanisms underlying the generation of metachronal rhythms in vivo are still only partially understood, their replication in silico may also enhance our understanding of this phenomenon. This topic of vibrating motor manipulation has been implemented in open loop systems ${ }^{19}$ and will be the subject of future work with this closed-loop platform. Local communication can be employed in future systems to organise a multitude of sorting algorithms, both bio-inspired or artificial.

A limitation of the board layout is the $15 \mathrm{~mm}$ distance between sensor and actuator, meaning that there may be an object on the actuator, it is not sensed unless it is above the sensor; this may account for the somewhat unsteady movement of the object. Currently the vibrating motor actuator only rotates in a single direction, leading to directional limitations, however improvements to the actuator will improve the level and direction of manipulation. The density of sensors and actuators is such that the object's diameter must be greater than $8 \mathrm{~cm}$ so that it is supported by at least 3 actuators; a smaller object and it may fall in between the actuators. MEMS based smart surfaces ${ }^{21}$ can manipulate much smaller and lighter objects (thin glass cover slips of a few $\mathrm{mm}$ in size in this example) but would ultimately fail to manipulate larger and heavier objects due to the lower force generated by the actuators; the density of sensor-actuator units may be improved in future generations. Zhou et al. 14 noted that MEMS cilia are markedly different from their biological counterpart, and vibrating motor actuators are no different; P. caudatum ciliary beat frequency is between 15 and $45 \mathrm{~Hz}{ }^{[22}$, compared to the vibrating motors' $205 \mathrm{~Hz}$. Future generations of MEMS or bespoke actuators may bridge the gap between bio-inspiration and become significantly more bio-mimetic.

\section{Conclusion}

We have presented a novel smart surface inspired by biological cilia for object manipulation. Distributed control is performed using tiled boards of sensor-actuators locally controlled by a microprocessor. We have demonstrated bidirectional object manipulation using autonomous closed-loop control using pairings of proximity sensors and vibrating motors. Analysis of the object tracking shows a mean object velocity of 7.3 millimetres per second in either direction. Further improvement to the actuators will likely produce more control over object manipulation. There are several limitations with 
the current system which we hope to address with ongoing research but this platform offers proof-of-concept for a scalable smart actuating surface with emergent properties.

We combined unconventional computing, bio-inspired engineering in a prototype of a parallel closed-loop actuators. In future developments, the actuators will be able to autonomously decide the particulars of orientation and transportation by itself without using any centralised facilities. The actuator array will intelligently recognise the object and will adapt to various shapes of manipulated objects; all using bio-inspired algorithms for localised neighbour communication. The manipulator surface will also process several objects at a time independently.

\section{Declaration of Interest}

The authors declare no conflict of interest and acknowledge the support of the Leverhulme Trust research project "Artificial P. caudatum: intelligent distributed sensing and manipulation by ciliates" (Number RPG-2013-345).

\section{References}

[1] D Mitchell. The evolution of eukaryotic cilia and flagella as motile and sensory organelles. Advances in experimental medicine and biology, 607:130-140, 2007.

[2] Sidney L. Tamm. Ciliary motion in Paramecium A Scanning Electron Microscope Study. The Journal of Cell Biology, 55(1):250-255, 1972.

[3] Inés Ibanez-Tallon, Nathaniel Heintz, and Heymut Omran. To beat or not to beat: roles of cilia in development and disease. Human Molecular Genetics, 12(suppl 1):R27-R35, 2003.

[4] P Satir and S Christensen. Structure and function of mammalian cilia. Histochemistry and Cell Biology, 129(6):687-693, 2008.

[5] Richard Mayne, James G.H. Whiting, Gabrielle Wheway, Chris Melhuish, and Andrew Adamatzky. Particle Sorting by Paramecium Cilia Arrays. Biosystems, 156-157:46-52, 2017. 
[6] Michael A Peshkin and Arthur C Sanderson. Planning robotic manipulation strategies for workpieces that slide. IEEE Journal on Robotics and Automation, 4(5):524-531, 1988.

[7] Patrick M McGuire. Conveyors: application, selection, and integration. CRC Press, 2009.

[8] B Borovic, AQ Liu, D Popa, H Cai, and FL Lewis. Open-loop versus closed-loop control of mems devices: choices and issues. Journal of Micromechanics and Microengineering, 15(10):1917, 2005.

[9] K.-F. Böhringer and Howie Choset. Distributed manipulation. Springer Science \& Business Media, 2012.

[10] Mikell P Groover. Automation, Production Systems, and ComputerIntegrated Manufacturing. Pearson Education Limited, third edition, 2014.

[11] K.Y. Goldberg. Orientating polygonal parts without sensors. Algorithmica, 10(2):201-225, 1993.

[12] Lydia E Kavraki. Part orientation with programmable vector fields: Two stable equilibria for most parts. In Robotics and Automation, $199 \%$. Proceedings., 1997 IEEE International Conference on, volume 3, pages 2446-2451. IEEE, 1997.

[13] K.-F. Bohringer, Bruce Randall Donald, and Noel C MacDonald. Programmable force fields for distributed manipulation, with applications to mems actuator arrays and vibratory parts feeders. The International Journal of Robotics Research, 18(2):168-200, 1999.

[14] Zhi guo Zhou and Zhi wen Liu. Biomimetic Cilia Based on MEMS Technology. Journal of Bionic Engineering, 5(4):358-365, 2008.

[15] Manabu Ataka, Bernard Legrand, Lionel Buchaillot, Dominique Collard, and Hiroyuki Fujita. Design, fabrication, and operation of two-dimensional conveyance system with ciliary actuator arrays. IEEE/ASME Transactions on Mechatronics, 14(1):119-125, 2009. 
[16] K. Boutoustous, G. J. Laurent, E. Dedu, L. Matignon, J. Bourgeois, and N. Le Fort-Piat. Distributed control architecture for smart surfaces. IEEE/RSJ 2010 International Conference on Intelligent Robots and Systems, IROS 2010 - Conference Proceedings, pages 2018-2024, 2010 .

[17] John W. Suh, R. Bruce Darling, Karl F. Bohringer, Bruce R. Donald, Henry Baltes, and Gregory T A Kovacs. CMOS integrated ciliary actuator array as a general-purpose micromanipulation tool for small objects. Journal of Microelectromechanical Systems, 8(4):483-496, 1999.

[18] K.-F. Bohringer, B.R. Donald, R. Mihailovich, and N.C. MacDonald. Sensorless manipulation using massively parallel microfabricated actuator arrays. Proceedings of the 1994 IEEE International Conference on Robotics and Automation, pages 826 - 833, 1994.

[19] Ioannis Georgilas, Andrew Adamatzky, David Barr, Piotr Dudek, and Chris Melhuish. Metachronal waves in cellular automata: Cilia-like manipulation in actuator arrays. Studies in Computational Intelligence, 512:261-271, 2014.

[20] P Brehm and R Eckert. An electrophysiological study of the regulation of ciliary beating frequency in paramecium. Journal of Physiology, 283:557-568, 1978.

[21] K. F. Bohringer, B. R. Donald, and N. C. MacDonald. Single-crystal silicon actuator arrays for micro manipulation tasks. Micro Electro Mechanical Systems, 1996, MEMS '96, Proceedings. An Investigation of Micro Structures, Sensors, Actuators, Machines and Systems. IEEE, The Ninth Annual International Workshop on, pages 7-12, Feb 1996.

[22] Anette Funfak, Cathy Fisch, T Abdel Motaal, Julien Diener, Laurent Combettes, N Baroud, and Pascale Dupuis-williams. Integrative Biology Paramecium swimming and ciliary beating patterns : a study on four RNA interference mutations. Integrative Biology, 2014. 